\title{
Efficacy and Safety of Combined Vitrectomy with Intravitreal Dexamethasone Implant for Advanced Stage Epiretinal Membrane
}

This article was published in the following Dove Press journal:

Drug Design, Development and Therapy

\author{
Claudio lovino $\left.{ }^{\prime}\right)^{\prime}$ \\ Giuseppe Giannaccare ${ }^{2}$ \\ Marco Pellegrini (D) ${ }^{2}$ \\ Federico Bernabei ${ }^{2}$ \\ Mirco Braghiroli ${ }^{1}$ \\ Tomaso Caporossi ${ }^{3}$ \\ Enrico Peiretti ${ }^{1,4}$ \\ 'Department of Surgical Sciences, Eye \\ Clinic, University of Cagliari, Cagliari, \\ Italy; ${ }^{2}$ Ophthalmology Unit, S. Orsola- \\ Malpighi University Hospital, University \\ of Bologna, Bologna, Italy; ${ }^{3}$ Department \\ of Ophthalmology, Ospedale Careggi, \\ Firenze, Italy; ${ }^{4}$ Clinica Oculistica, San \\ Giovanni di Dio Hospital, Azienda \\ Ospedaliera Universitaria di Cagliari, \\ Cagliari, Italy
}

Purpose: To evaluate the efficacy and safety of combined 25-gauge $(\mathrm{G})$ pars plana vitrectomy (PPV) with intravitreal dexamethasone implant (DXI) for the treatment of advanced stage epiretinal membrane (ERM).

Methods: Forty consecutive pseudophakic eyes with idiopathic stage 3-4 ERM and intraretinal cysts were randomly assigned to two treatment groups. Twenty eyes underwent combined 25-G PPV, ERM peeling and slow-release DXI (DEX group), whereas 20 eyes underwent standard 25-G PPV with ERM peeling only (control group). Differences in postoperative best-corrected visual acuity (BCVA), intraocular pressure (IOP), central macular thickness (CMT) were evaluated.

Results: In all patients, BCVA significantly increased at 1, 3 and 6 months after surgery compared to baseline (all $p<0.05$ ), but at 3 and 6 months, the visual gain was higher in the DEX group (respectively, $p=0.036, p=0.006$ ). CMT was significantly lower in DEX group compared to control group at 3 and 6 months after surgery (respectively, $p=0.042, p=$ $0.003)$. There was no statistically significant difference in IOP change over the course of the follow-up between groups $(p>0.05)$.

Conclusion: Combined 25-G PPV with DXI is associated with better anatomical and functional outcomes in patients with advanced stage ERM.

Keywords: epiretinal membrane, intravitreal dexamethasone, macular pucker, intraretinal cysts

\section{Introduction}

Epiretinal membrane (ERM) is a common retinal disorder of the vitreoretinal interface, significantly associated with aging. ${ }^{1,2}$ Decrease in visual acuity, with or without metamorphopsia secondary to morphologic distortions of the retinal surface, represents the main indication for ERM surgery. ${ }^{3}$ Standard surgical treatment consists of pars plana vitrectomy (PPV) and ERM removal resulting in good visual recovery. ${ }^{4}$

Macular edema can be a cause of visual loss in eyes undergoing vitrectomy with ERM peeling, and its incidence evaluated by optical coherence tomography (OCT) is up to $47 \% .^{5}$ In the multifactorial pathogenesis of post-surgery cystoid macular edema (CME), inflammation plays an important role. Moreover, eyes with preoperative intraretinal cysts have a higher probability to develop CME after the surgery. ${ }^{6}$ Many treatments have been proposed for post-surgical CME, including ocular nonsteroidal anti-inflammatory drugs and systemic, periocular or intravitreal corticosteroids for their potential anti-inflammatory properties. 5,7
Correspondence: Enrico Peiretti Eye Clinic, University of Cagliari, Via Ospedale 48, Cagliari 09124, Italy TellFax +390706092319

Email enripei@hotmail.com 
Recently, some authors reported the efficacy of intravitreal dexamethasone implant (DXI) for the treatment of postoperative CME after macular pucker removal alone ${ }^{8}$ or combined with cataract surgery. ${ }^{9}$ Supporting the hypothesis of a possible inflammatory etiology, Suzuki et a ${ }^{10}$ postulated that ERM traction stimulates a leukocyte response in the macular region causing persistent macular edema even after its surgical removal.

Based on this concept, the aim of the present study was to prospectively investigate the efficacy and safety of combined 25-gauge (G) PPV with intraoperative DXI for the treatment of advanced stage ERM with intraretinal cysts.

\section{Methods}

This was a prospective randomized controlled study conducted at the Retina Center of the Eye Clinic, University of Cagliari, Italy. The investigation was approved by the Office of Research Ethics, University of Cagliari and performed according to the guidelines of the Declaration of Helsinki and to the recommendation described in the Consolidated Standard of Reporting Trials (CONSORT) Statement.

\section{Patients and Clinical Examination}

Pseudophakic patients (operated at least 6 months before the enrollment) with an idiopathic stage 3-4 ERM defined on the basis of a recent spectral-domain optical coherence tomography (SD-OCT) classification and intraretinal cysts were included in the study. ${ }^{11}$ Phakic eyes were excluded to reduce any possible bias for postoperative analysis. ERM was visualised on OCT, as irregular and hyperreflective bands above the inner retinal surface. A stage 3 ERM was defined in case of absence of the foveal pit, presence of ectopic inner foveal layer (EIFL) and well-defined retinal layers. Stage 4 ERM was assigned to all eyes with the absence of foveal pit, presence of EIFL and disrupted retinal layers. Exclusion criteria are summarized in Table 1.

Patients were randomly assigned into two groups: one receiving the combined 25-G PPV with ERM peeling plus DXI (DEX group), and one undergoing 25-G PPV with ERM peeling alone (control group). All patients who received the DEX implant were aware of its off-label use and provided written informed consent.

The randomization process was computer generated with a 1:1 ratio. Follow-up visits were scheduled at 1 and 7 days and 1, 3 and 6 months after the surgery and included: best-corrected visual acuity (BCVA) reported in Snellen fraction with minimal angle of resolution
Table I Study of Intravitreal Dexamethasone Implant for the Treatment of Advanced Stage Epiretinal Membrane: Exclusion Criteria

- Age-related macular degeneration

- History of choroidal neovascularization of any etiology

- Nonproliferative and proliferative diabetic retinopathy

- Severe myopia (>6 diopters or axial length $>25 \mathrm{~mm}$ )

- Macular telangiectasia

- Retinal dystrophy

- Central serous chorioretinopathy

- History of uveitis or inflammatory eye disorders (including IrvineGass Syndrome)

- History of any intraocular infection

- History of corneal herpetic infection

- History of central or branch retinal vein and/or artery occlusion

- Glaucoma or optic neuropathy of any kind

- Associated lamellar macular holes

- History of retinal detachment and any previous intraocular surgery with the exclusion of uncomplicated phacoemulsification

- Amblyopia or any other potential cause of vision loss other than epiretinal membrane in the study eye

( $\log$ MAR) conversion for statistical analysis, intraocular pressure (IOP) measurement with Goldmann applanation tonometry, slit-lamp and fundus examination and SD-OCT analysis (SD-OCT, Heidelberg Spectralis HRA + OCT, Heidelberg Engineering, Germany). The "follow-up" function of the Heidelberg OCT was used to ensure the same macular area was evaluated at each visit.

The OCT recording protocol consisted of a sequence of 49 horizontal sections covering an area of 20 degree. Central macular thickness (CMT) was obtained with the automatic "thickness map" function of the Heidelberg Eye Explorer. Outer nuclear layer (ONL) and EIFL thickness were manually measured with the caliper function of the Heidelberg instrument. The presence of intraretinal cystoid spaces along with the integrity of the ellipsoid zone and external limiting membrane in the macular zone were recorded. Any discontinuity or interruption of these latter was considered as an alteration. All imaging data were collected and analyzed by one examiner (CI), then selectively reviewed by the senior author (EP) to ascertain all retinal findings.

\section{Surgical Procedure}

All patients were operated by the same surgeon (EP) under local anesthesia with retrobulbar lidocaine injection. They underwent a standard three-port sutureless 25-G PPV using the Constellation vitrectomy system (Alcon Laboratories). After core vitrectomy, a peripheral vitreous 
shaving with a scleral indentation in every clock hour was also performed. Whenever required, a posterior vitreous detachment was inducted using the vitrectomy probe in the suction mode around the optic nerve disc. The ERM was peeled with intraocular forceps until the vascular arcades after $0.1 \mathrm{~mL}$ of Membraneblue-dual (TrypanBlue $0.15 \%+$ Brilliant Blue $0.025 \%$ + PEG 4\%, DORC, Zuidland, The Netherlands) coloration for approximately $1 \mathrm{~min}$ after stopping the infusion.

The ILM was removed routinely in both groups, either at the same time or after the ERM removal up to two-three disk diameters centered on the fovea. Second staining was performed to ascertain the complete removal of the ILM in the intended area only in case it was not clearly evident. A detailed examination of the periphery was conducted during surgery to ascertain the absence of any retinal holes or tears. At the end of the surgery, a fluid-air exchange procedure was done for all study eyes followed by trocars removal. In the DEX group patients, a $0.7 \mathrm{mg}$ sustainedrelease DXI (Ozurdex ${ }^{\circledR}$, Allergan, Inc., Irvine, CA, USA and Allergan Pharmaceuticals, Ireland) was cautiously injected under air through the superotemporal scleral port with a gentle force applied on the injector gradually to avoid any projectile damage to the retina. The implant position was confirmed at the end of the procedure by direct visualization through the indirect operating microscope. Postoperative medication consisted of topical antibiotic-corticosteroid association tapered and completely withdrawn at 4 weeks after surgery.

\section{Statistical Analysis}

The SPSS statistical software (SPSS Inc, Chicago, Illinois, USA) was used for data analysis. Values are expressed as mean \pm standard deviation (SD). An independent-samples $t$-test was used to compare continuous variables between groups. A $\chi^{2}$ test was used to compare dichotomous variables between groups. A repeated-measures ANOVA was used to determine whether there was a statistically significant change in clinical variables over the course of the 6-month follow-up after surgery. A mixed ANOVA was used to determine whether any change in clinical variables was the result of the interaction between the type of intervention and time. The Shapiro-Wilk's test was used to assess the normality of data.

The Levene's test and the Box's M test were used to assess the homogeneity of variances and covariances, respectively. For the interaction between the type of intervention and time on BCVA, CMT, ONL thickness, EIFL thickness and IOP, the assumption of sphericity was violated, as assessed by Mauchly's test (all $p<0.05$ ). Therefore, a Greenhouse-Geisser correction was applied. A $p$ value $<0.05$ was considered statistically significant.

A power analysis indicated that a minimum sample of 24 patients was required to detect a $0.017 \log$ MAR difference in terms of BCVA between groups, with a power of $80 \%$ and a significance level of $0.05 .^{12}$

\section{Results}

\section{Study Population}

Overall, a total of 89 patients were recruited for eligibility and 40 met the inclusion criteria (Figure 1). Baseline and clinical characteristics of study patients are reported in Table 2 .

No significant differences in age, sex, ERM stage, IOP, EZ and ELM integrity were found between the two groups (all $p>0.05$ ). No intra or post-operative complications were recorded in either group.

No patient of the ones who met the inclusion criteria dropped out from the study.

In both groups, BCVA significantly improved at 1, 3 and 6 months after surgery compared to baseline (all $p<0.05$, Table 3 ).

There was a statistically significant difference in the change of BCVA over the course of the follow-up between groups $(\mathrm{F}=17.050 ; p<0.001)$.

BCVA was not significantly different in DEX group compared to control group at baseline $(p=0.702)$ and 1 month after surgery $(p=0.198)$. Conversely, it was significantly better in DEX implant group compared to control group at 3 months $(p=0.036)$, continuing to improve at the 6-month post-operative visit $(p=0.006)$.

In both groups, CMT significantly decreased at 1, 3 and 6 months after surgery compared to baseline (all $p<0.05$, Table 3). There was a statistically significant difference in the change of central macular thickness over the course of the follow-up between groups $(\mathrm{F}=9.143 ; p=0.001)$. In particular, the CMT was not significantly different in the DEX group compared to the control group at baseline $(p=0.171)$ and 1 month after surgery $(p=0.688)$. Conversely, it was significantly lower in DEX implant group compared to control group at 3 months $(p=0.042)$ and 6 months after surgery $(p=0.003)$.

In both groups, ONL thickness significantly decreased at 1 , 3 and 6 months after surgery compared to baseline (all $p<0.05$, Table 3 ). There was no statistically significant difference in the measurement of ONL thickness over the course of the followup between groups $(\mathrm{F}=0.656 ; p=0.485)$. 


\section{Enrollment}

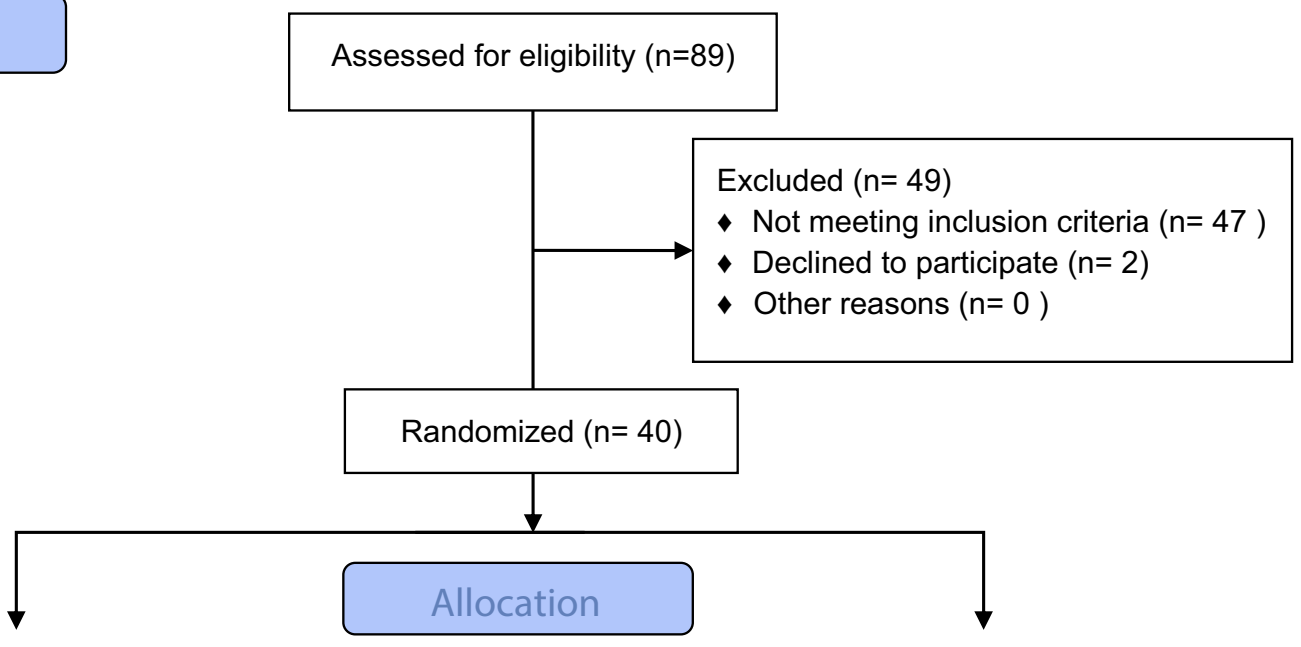

\begin{tabular}{|c|c|c|}
\hline $\begin{array}{l}\text { DEX Group } \\
\text { Allocated to intervention }(n=20) \\
\text { Received allocated intervention }(n=20) \\
\text { Did not receive allocated intervention (give } \\
\text { reasons) }(n=0)\end{array}$ & & $\begin{array}{l}\text { Control Group } \\
\text { Allocated to intervention }(n=20) \\
\text { Received allocated intervention }(n=20) \\
\text { Did not receive allocated intervention (give } \\
\text { reasons) }(n=0)\end{array}$ \\
\hline & \multirow[t]{2}{*}{ Follow-Up } & $\downarrow$ \\
\hline $\begin{array}{l}\text { Lost to follow-up (give reasons) }(n=0) \\
\text { Discontinued intervention }(n=0)\end{array}$ & & $\begin{array}{l}\text { Lost to follow-up }(n=0) \\
\text { Discontinued intervention }(n=0)\end{array}$ \\
\hline$\downarrow$ & \multirow[t]{2}{*}{ Analysis } & $\downarrow$ \\
\hline $\begin{array}{l}\text { Analysed }(n=20) \\
\text { Excluded from analysis }(n=0)\end{array}$ & & $\begin{array}{l}\text { Analysed }(n=20) \\
\text { Excluded from analysis }(n=0)\end{array}$ \\
\hline
\end{tabular}

Figure I Recruitment Flowchart.

In the DEX group, EIFL thickness significantly decreased at 1, 3 and 6 months after surgery compared to baseline (all $p<0.05$, Table 3), while in the control group, EIFL thickness did not significantly change (all $p>0.05$, Table 3). There were no statistically significant differences in the change of EIFL over the course of the follow-up between groups $(\mathrm{F}=1.710, p=0.195)$.

In the DEX group, IOP significantly increased at 1 month after surgery compared to baseline (from $14.7 \pm$ 2.1 to $18.0 \pm 1.5 \mathrm{mmHg}, p<0.001)$. However, it returned to values not significantly different from baseline at 3 and 6 months after surgery (respectively, $16.0 \pm 2.6 \mathrm{mmHg}$, $p=0.809 ; 15.4 \pm 2.4 \mathrm{mmHg}, p=1.000)$. In the control group, IOP did not significantly change at 1,3 and 6 months after surgery (all $p>0.05$ ). There were no statistically significant differences in the change of IOP over the course of the follow-up between groups $(\mathrm{F}=1.970 ; p=$ 0.095).

Six months after surgery, only one patient $(5.0 \%)$ in the DEX group had intraretinal cysts, while eight patients $(40.0 \%)$ in the control group did $(p=0.008)$. A representative case is shown in Figure 2.

Conversely, no significant differences in the number of patients with EZ disruption and ELM disruption were found between DEX group and control group (respectively, 10.0\% vs $15.0 \%, p=0.633 ; 15.0 \%$ vs $15.0 \%, p=1.000$ ). 
Table 2 Demographical and Clinical Characteristics of Patients in Dexamethasone Group and Control Group Before Surgery

\begin{tabular}{|l|l|l|l|}
\hline & DEX Group $(\mathbf{n}=\mathbf{2 0})$ & Control Group $(\mathbf{n}=\mathbf{2 0})$ & $P$ \\
\hline Age (years) & $74.2 \pm 6.0$ & $70.1 \pm 9.2$ & 0.136 \\
Sex (m/f) & $8 / 12$ & $7 / 13$ & 0.744 \\
ERM stage (stage 3/4) & $9 / 11$ & $7 / 13$ & 0.519 \\
IOP (mmHg) & $14.7 \pm 2.1$ & $15.1 \pm 2.0$ & 0.550 \\
Intraretinal cysts & $20(100.0 \%)$ & $20(100.0 \%)$ & 1.000 \\
EZ disruption (\%) & $8(40.0 \%)$ & $5(25.0 \%)$ & 0.311 \\
ELM disruption (\%) & $7(35.0 \%)$ & $3(15.0 \%)$ & 0.144 \\
\hline
\end{tabular}

Abbreviations: ERM, epiretinal membrane; IOP, intraocular pressure; EZ, ellipsoid zone; ELM, external limiting membrane.

Table 3 Best Corrected Visual Acuity, Central Macular Thickness, Outer Nuclear Layer Thickness and Ectopic Inner Foveal layer Thickness in Dexamethasone Group and Control Group Over the Course of the 6-Month Follow-Up

\begin{tabular}{|c|c|c|c|}
\hline & DEX Group & Control Group & $P^{\#}$ \\
\hline \multicolumn{4}{|c|}{ Best corrected visual acuity (logMAR, Snellen) } \\
\hline Baseline & $0.69 \pm 0.32(20 / 100)$ & $0.65 \pm 0.26(20 / 90)$ & 0.702 \\
\hline I month & $0.43 \pm 0.27^{*}(20 / 55)$ & $0.55 \pm 0.24 *(20 / 70)$ & 0.198 \\
\hline 3 months & $0.32 \pm 0.23 *(20 / 40)$ & $0.46 \pm 0.23^{*}(20 / 60)$ & 0.036 \\
\hline 6 months & $0.26 \pm 0.23 *(20 / 35)$ & $0.41 \pm 0.22 *(20.50)$ & 0.006 \\
\hline \multicolumn{4}{|c|}{ Central macular thickness $(\mu \mathrm{m})$} \\
\hline Baseline & $567.3 \pm 118.4$ & $522.7 \pm 71.9$ & 0.171 \\
\hline I month & $446.2 \pm 99.1 *$ & $450.6 \pm 65.4^{*}$ & 0.688 \\
\hline 3 months & $374.8 \pm 64.5^{*}$ & $420.6 \pm 64.4^{*}$ & 0.042 \\
\hline 6 months & $343.1 \pm 70.6^{*}$ & $4 \mid 4.6 \pm 62.0 *$ & 0.003 \\
\hline \multicolumn{4}{|c|}{ Outer nuclear layer thickness $(\mu \mathrm{m})$} \\
\hline Baseline & $270.9 \pm 101.5$ & $254.1 \pm 70.8$ & 0.561 \\
\hline I month & $217.8 \pm 50.1 *$ & $222.4 \pm 50.0^{*}$ & 0.788 \\
\hline 3 months & $194.9 \pm 36.2^{*}$ & $198.6 \pm 54.0^{*}$ & 0.817 \\
\hline 6 months & $191.8 \pm 35.5^{*}$ & $197.3 \pm 55.2^{*}$ & 0.730 \\
\hline \multicolumn{4}{|c|}{ Ectopic inner foveal layer thickness $(\mu \mathrm{m})$} \\
\hline Baseline & $223.4 \pm 123.8$ & $183.0 \pm 103.8$ & 0.294 \\
\hline I month & $189.4 \pm 93.1 *$ & $181.8 \pm 78.1$ & 0.960 \\
\hline 3 months & $138.8 \pm 84 .\left.\right|^{*}$ & $138.8 \pm 59.5$ & 0.998 \\
\hline 6 months & $130.6 \pm 86.6^{*}$ & $124.0 \pm 46.9$ & 0.773 \\
\hline
\end{tabular}

Notes: ${ }^{*} p<0.05$ compared to baseline, Tukey post hoc test for repeated-measures ANOVA. "DEX group compared to control group, one-way ANOVA.

\section{Discussion}

Epiretinal membrane is a fibrocellular preretinal tissue composed by fibroblasts, glial cells and hyalocytes over the ILM. ${ }^{13}$ Pars plana vitrectomy with pucker removal remains the standard treatment, albeit CME is a common source of postoperative visual limitation. ${ }^{5}$ In the present randomized controlled trial, we investigated the safety and efficacy of intravitreal DXI combined with $25 \mathrm{G}$ vitrectomy for advanced stage ERM removal. The use of DXI was associated with a better visual outcome and a lower CMT at 3 and 6 months after surgery.

An inflammation component may co-exist and be a contributory cause of intraretinal fluid accumulation either before or after the surgery. Indeed, the mechanical distortion of the retinal surface induced by ERM traction stimulates an inflammation pathway involving different growth factors and cytokines, with leukocyte migration in the macular region. ${ }^{10}$ This biochemical process contributes to a diffuse retinal thickening associated in some cases to the formation of CME.

Azzolini and associate analyzed the composition of the ERM by means of scanning electron microscopy, reporting four types of structures from the ILM to the vitreous side. ${ }^{14}$ The layer facing towards the vitreous was defined as a lacunar structure with inflammatory material. On the other hand, inflammation plays an important role also in CME occurring after ERM surgical removal. Its pathogenesis is attributed to the breakdown of the blood-aqueous barrier caused by the inflammatory surgery-related reaction. ${ }^{15}$ Based on these concepts, corticosteroids may be able to reduce the inflammatory events related to the mechanical distortion caused by ERM and to the surgical insult. Chatziralli and coauthors investigated the long-term outcomes of DXI for the treatment of macular edema following ERM surgical removal demonstrating its safety and efficacy. ${ }^{12}$ Likewise, Furino et al demonstrated the efficacy of a single DXI in refractory macular edema secondary to combined cataract extraction and vitrectomy for ERM removal. ${ }^{9}$

The data about the intraoperative use of DXI are controversial. In a case-control study, intraoperative intravitreal corticosteroids, both DXI and triamcinolone acetonide, showed to accelerate the normalization of macular morphology and were effective in improving visual and anatomic outcomes in patients with macular pucker. ${ }^{16}$ More recently, Guidi and associate reported no advantages in terms of BCVA and 


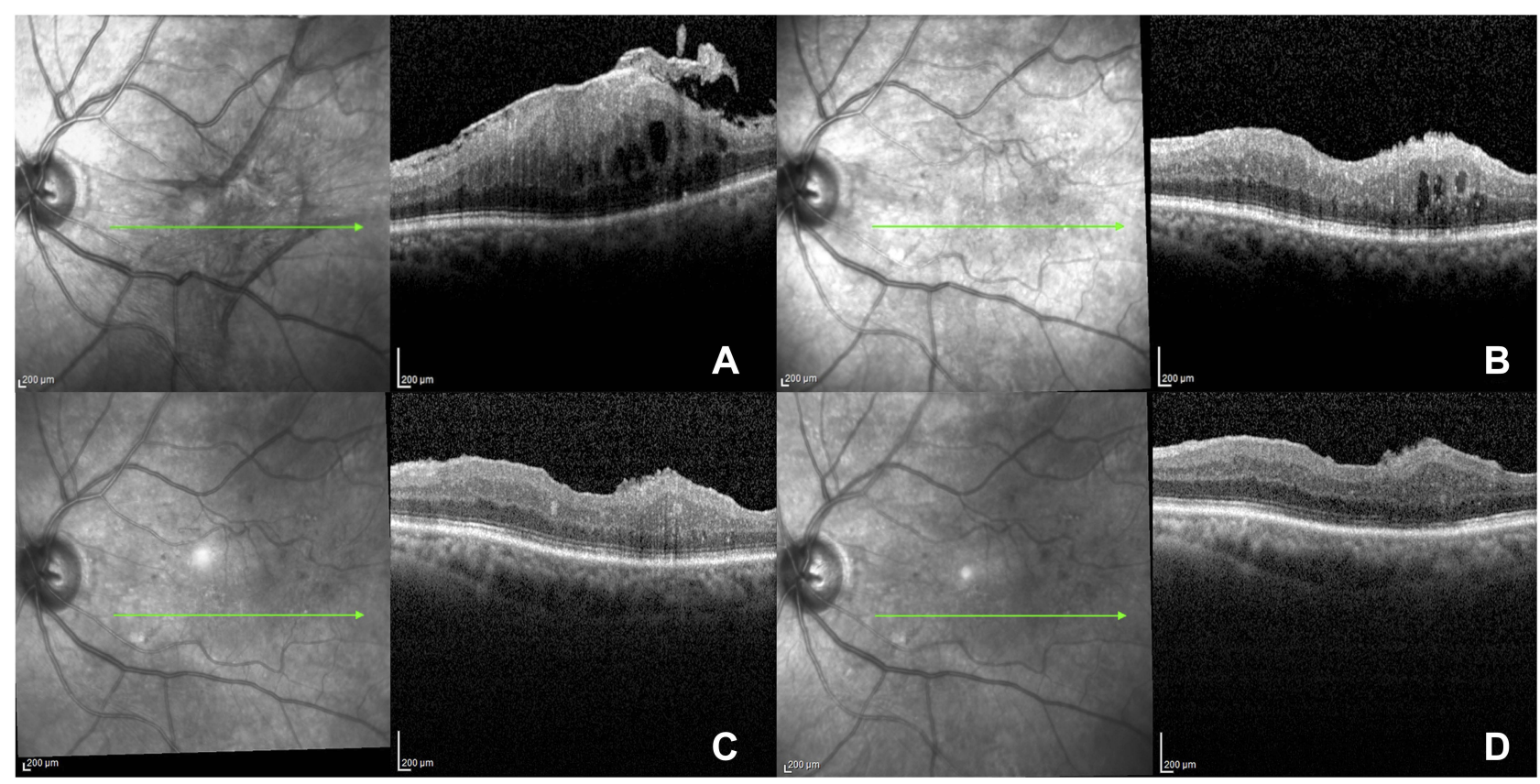

Figure 2 Preoperative and Postoperative SD-OCT scans of patients with stage 4 ERM and intraretinal cysts undergoing $25 \mathrm{G}$ VVP plus intraoperative DEX implant. (A) Baseline stage 4 ERM with intraretinal cysts. (B) At I month after surgery, despite significant retinal thinning, few cysts are present. (C) At 3 months after surgery, intraretinal cystoid changes completely resolved. (D) At 6 months after surgery, the clinical picture remains stable. A good anatomical restoration is achieved.

CMT for patients receiving an intraoperative sustained-release DXI, although they did not make any differences neither for macular pucker stage nor for the presence/absence of intraretinal cysts. ${ }^{17}$ In our study, only patients with stage 3-4 ERM with intraretinal cysts were enrolled, in which the use of intraoperative DXI has a stronger clinical rationale.

The prevalence of CME increases according to the ERM stage, ${ }^{11}$ due to the stronger tractional forces producing retinal layer disruption with subsequent interstitial fluid accumulation. Moreover, the thickness of inner retinal tissue above the fovea referred to as EIFL is negatively correlated with the visual acuity. ${ }^{18}$

The pathophysiological mechanism explaining this association is still unknown, but it could be related to the interference between the afferent light and the overlaying foveal ectopic tissue in a zone of high cone specialization. In the DEX group, EIFL thickness significantly decreased at 1,3 and 6 months after surgery compared to baseline as a possible result of the reduction/inhibition of reactive gliosis promoted by the corticosteroids.

The reduction in CMT and EIFL thickness may explain the better visual acuity in patients receiving intraoperative DXI. In support of this hypothesis, Kim et al reported a more rapid visual recovery in eyes showing a greater decrease in CMT after vitrectomy. ${ }^{5}$
The safety and efficacy of $0.7 \mathrm{mg}$ DXI in vitrectomized eyes has been proved in several studies. ${ }^{19,20}$ The most frequent ophthalmic adverse effect is the transient elevation of IOP, managed medically in up to $95 \%$ of cases. ${ }^{21}$ In DEX group patients, IOP significantly increased at 1 month after surgery compared to baseline but no anti-glaucoma medication was needed in any patient. Additionally, no statistically significant differences in the change of IOP were recorded between groups over the course of the follow-up $(\mathrm{F}=1.970 ; p=0.095)$.

Regarding the real advantage of the ILM peeling on BCVA in macular pucker surgery, it is still controversial, but a recent meta-analysis proved that it could reduce the recurrence rate of ERM. ${ }^{22}$ Following this evidence and to minimize any potential bias between groups, the ILM was removed in all study eyes. As known, this may cause damage to the Muller cells and it could explain the cases with persistent intraretinal cystoid spaces.

The main limitations of this study included the small population, the lack of fluorescein angiography to characterize the CME and the relatively short follow-up period. Larger, prospective and controlled studies are needed to confirm our hypothesis. Moreover, the readers (CI, EP) were not masked to the treatment groups, and therefore ascertainment bias may have influenced an accurate interpretation of the results. 
An additional study limitation is the absence of a third arm receiving triamcinolone acetonide to ascertain any similar effect at a lower cost. However, the clearance of the intravitreal triamcinolone is relatively fast in vitrectomized eyes, ${ }^{23}$ and we believe DXI may, therefore, be advantageous providing a slow-release and steady dose of potent steroid.

In conclusion, this report provides evidence of the efficacy and safety of combined $25 \mathrm{G}$ vitrectomy with DXI procedure in patients with advanced stage ERM and intraretinal cysts. The anti-inflammatory properties of the intraocular dexamethasone in addition to vitrectomy and ERM peeling can be very useful in stage 3 and 4 ERM where an increased traction component exists.

\section{Acknowledgments}

This work was supported by the Open Access Publishing Fund of the University of Cagliari, with the funding of the Regione Autonoma della Sardegna - L.R. n. 7/2007.

\section{Disclosure}

The authors report no conflicts of interest in this work.

\section{References}

1. $\mathrm{Ng} \mathrm{CH}$, Cheung N, Wang JJ, et al. Prevalence and risk factors for epiretinal membranes in a multi-ethnic United States population. Ophthalmology. 2011;118(4):694-699. doi:10.1016/j.ophtha.2010.08.009

2. Mitchell P, Smith W, Chey T, Wang JJ, Chang A. Prevalence and associations of epiretinal membranes: the blue mountains eye study, Australia. Ophthalmology. 1997;104(6):1033-1040. doi:10.1016/ S0161-6420(97)30190-0

3. Smiddy WE, Michels RG, Green WR. Morphology, pathology, and surgery of idiopathic vitreoretinal macular disorders. A review. Retina. 1990;10(4):288-296. doi:10.1097/00006982-199010000-00012.

4. Miliatos I, Lindgren G. Epiretinal membrane surgery evaluated by subjective outcome. Acta Ophthalmol. 2017;95(1):52-59. doi:10.1111/aos.13001

5. Kim SJ, Martin DF, Hubbard GB, et al. Incidence of postvitrectomy macular edema using optical coherence tomography. Ophthalmology. 2009;116(8):1531-1537. doi:10.1016/j.ophtha.2009.02.008

6. Frisina R, Pinackatt SJ, Sartore M, et al. Cystoid macular edema after pars plana vitrectomy for idiopathic epiretinal membrane. Graefe's Arch Clin Exp Ophthalmol. 2014;253(1):47-56. doi:10.1007/s00417-014-2655-x

7. Johnson MW. Etiology and treatment of macular edema. $\mathrm{Am}$ J Ophthalmol. 2009;147(1):11-21.e1. doi:10.1016/j.ajo.2008.07.024

8. Hattenbach LO, Springer-Wanner C, Hoerauf H, et al. Intravitreal sustained-release steroid implants for the treatment of macular edema following surgical removal of epiretinal membranes. Ophthalmologica. 2017;237(4):232-237. doi:10.1159/000464259
9. Furino C, Boscia F, Recchimurzo N, Sborgia C, Alessio G. Intravitreal dexamethasone implant for refractory macular edema secondary to vitrectomy for macular pucker. Retina. 2014;34 (8):1612-1616. doi:10.1097/IAE.0000000000000105

10. Suzuki T, Hayakawa K, Nakagawa Y, Onouchi H, Ogata M, Kawai K. Topical dorzolamide for macular edema in the early phase after vitrectomy and epiretinal membrane removal. Clin Ophthalmol. 2013;7:549-553. doi:10.2147/OPTH.S42188

11. Govetto A, Lalane RA, Sarraf D, Figueroa MS, Hubschman JP. Insights into epiretinal membranes: presence of ectopic inner foveal layers and a new optical coherence tomography staging scheme. Am J Ophthalmol. 2017;175:99-113. doi:10.1016/j.ajo.2016.12.006

12. Chatziralli I, Dimitriou E, Theodossiadis G, Chatzirallis A, Kazantzis D, Theodossiadis P. Treatment of macular edema after pars plana vitrectomy for idiopathic epiretinal membrane using intravitreal dexamethasone implant: long-term outcomes. Ophthalmologica. 2019;1-6. doi:10.1159/ 000496705

13. Compera D, Entchev E, Haritoglou C, et al. Lamellar hole-associated epiretinal proliferation in comparison to epiretinal membranes of macular pseudoholes. Am J Ophthalmol. 2015;160(2):373-384.e1. doi:10.1016/j.ajo.2015.05.010

14. Azzolini C, Congiu T, Donati S, et al. Multilayer microstructure of idiopathic epiretinal macular membranes. Eur J Ophthalmol. 2017;27 (6):762-768. doi:10.5301/ejo.5000982

15. Tso MO. Pathology of cystoid macular edema. Ophthalmology. 1982;89(8):902-915. doi:10.1016/S0161-6420(82)34698-9.

16. Yonekawa Y, Mammo DA, Thomas BJ, Wolfe JD, Hassan TS. A comparison of intraoperative dexamethasone intravitreal implant and triamcinolone acetonide used during vitrectomy and epiretinal membrane peeling: a case control study. Ophthalmic Surgery, Lasers Imaging Retin. 2016;47(3):232-237. doi:10.3928/23258160-20160229-05

17. Guidi G, Casini G, Ripandelli G, et al. Residual intraretinal edema after 25-gauge vitrectomy and macular pucker removal: is intraoperative sustained-release dexamethasone a real treatment option? Retina. 2017:993-999. doi:10.1097/IAE.0000000000001627.

18. Song SJ, Lee MY, Smiddy WE. Ganglion cell layer thickness and visual improvement after epiretinal membrane surgery. Retina. 2016;36(2):305-310. doi:10.1097/IAE.0000000000000705

19. Boyer DS, Faber D, Gupta S, et al. Dexamethasone intravitreal implant for treatment of diabetic macular edema in vitrectomized patients. Retina. 2011;31(5):915-923. doi:10.1097/IAE.0b013e318206d18c

20. Dutra Medeiros M, Alkabes M, Navarro R, Garcia-Arumí J, Mateo C, Corcóstegui B. Dexamethasone intravitreal implant in vitrectomized versus nonvitrectomized eyes for treatment of patients with persistent diabetic macular edema. J Ocul Pharmacol Ther. 2014;30(9):709-716. doi:10.1089/jop.2014.0010

21. Rajesh B, Zarranz-Ventura J, Fung AT, et al. Safety of 6000 intravitreal dexamethasone implants. Br J Ophthalmol. 2019:bjophthalmol2019-313991. doi:10.1136/bjophthalmol-2019-313991.

22. Azuma K, Ueta T, Eguchi S, Aihara M. Effects of internal limiting membrane peeling combined with removal of idiopathic epiretinal membrane a systematic review of literature and meta-analysis. Retina. 2017:1-7. doi:10.1097/IAE.0000000000001537

23. Chin HS, Park TS, Moon YS, Oh JH. Difference in clearance of intravitreal triamcinolone acetonide between vitrectomized and nonvitrectomized eyes. Retina. 2005;25(5):556-560. doi:10.1097/ 00006982-200507000-00002 


\section{Publish your work in this journal}

Drug Design, Development and Therapy is an international, peerreviewed open-access journal that spans the spectrum of drug design and development through to clinical applications. Clinical outcomes, patient safety, and programs for the development and effective, safe, and sustained use of medicines are a feature of the journal, which has also been accepted for indexing on PubMed Central. The manuscript management system is completely online and includes a very quick and fair peer-review system, which is all easy to use. Visit http://www. dovepress.com/testimonials.php to read real quotes from published authors. 